
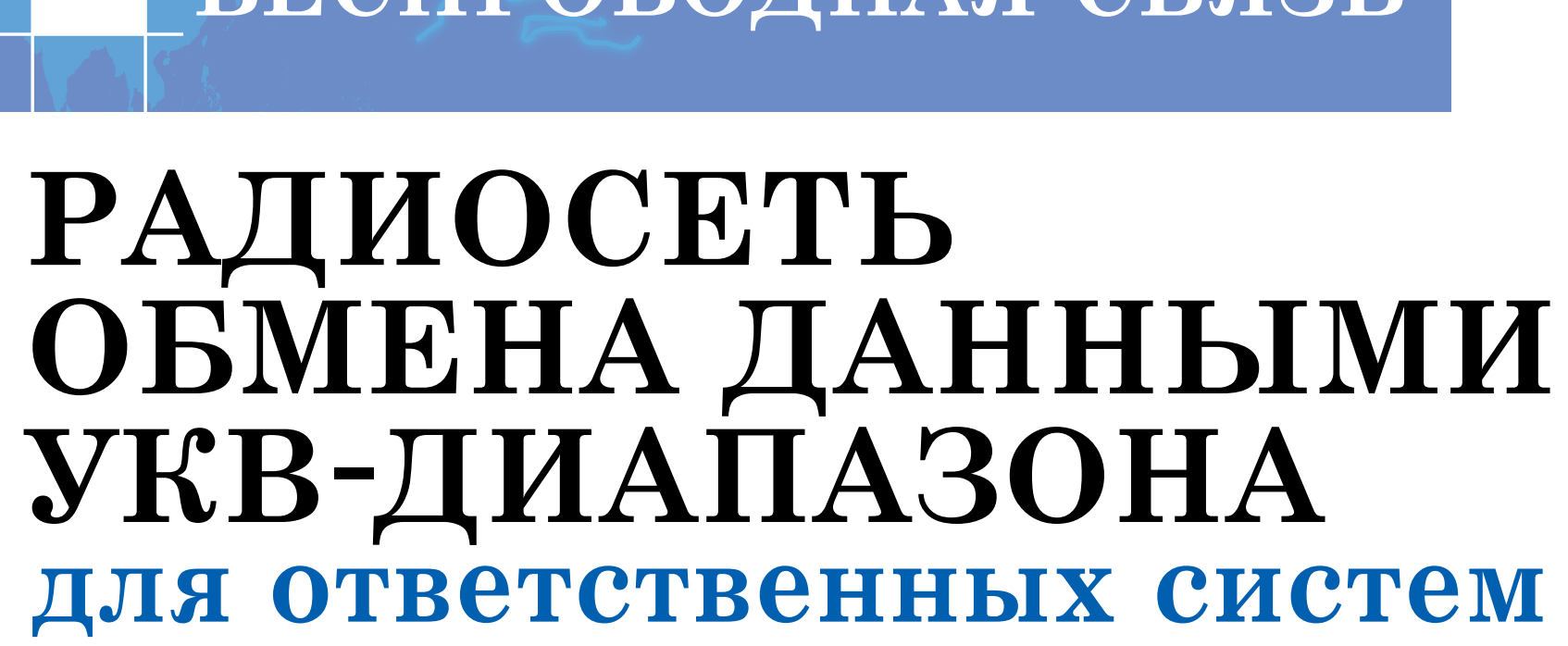

Yacmb 2

\author{
С.Маргарян, заместитель генерального директора, \\ главный конструктор ЗАО "НПП "Родник" / sm@rodnik.ru, \\ В.Осьмов, заместитель начальника отдела специальных проектов \\ ЗАО "НПП "Родник" / osmov@rodnik.ru, \\ В.Мостыко, технический директор \\ ООО "Профессиональные радиосистемы" / Slava.Mostuko@prs.by
}

УДК 654.16, DOI: 10.22184/2070-8963.2017.65.4.58.61

Описаны особенности построения и функциональные возможности узкополосных технологических радиосетей обмена данными УКВ-диапазона повышенной надежности и живучести для ответственных систем. Дано краткое сравнение их с проводными сетями аналогичного назначения.

\section{ИНФОРМАЦИОННАЯ СИСТЕМА}

Уровни надежности и живучести описанных выше вариантов реализации БС значительно превышают аналогичные параметры для типовых вычислительных средств. Параметры надежности и живучести вычислительных средств могут быть существенно улучшены за счет применения специального ПО Stratus everRun, защищаюеего приложения от отказов и сбоев в работе аппаратной части сервера. Это ПО позволяет организовать непрерывное выполнение приложений благодаря созданию общей операционной среды из двух физических серверов с использованием виртуализации. Stratus everRun полностью дублирует окружение прикладной программы, включая данные в памяти, гарантируя работу без потерь и прерываний. В случае, если один из физических узлов перестает работать, приложение продолжает выполняться на другом узле. При отказе аппаратного компонента ПО подменяет его работоспособным компонентом второго узла до тех пор, пока вышедший из строя компонент не будет восстановлен или заменен.
В качестве технической основы используется вычислительный комплекс повышенной надежности и живучести в составе двух идентичных вычислительных узлов, каждый из которых имеет собственное дисковое пространство. Узлы связаны друг с другом сетевыми интерфейсами. Защищаемые приложения запущены на виртуальном сервере. Упрощенная схема вычислительного комплекса повышенной надежности и живучести под управлением ПО Stratus everRun представлена на рис.7.

ПО everRun устанавливается на оба сервера в несколько шагов, основная настройка происходит автоматически. Виртуальные машины создаются и управляются через встроенный web-интерфейс.

Реакция системы на сбой определяется выбранным уровнем отказоустойчивости: первый - начальный; второй - на уровне компонентов; третий - полная отказоустойчивость.

На первом уровне при выходе из строя одного из узлов или его компонентов, защищаемая OC 


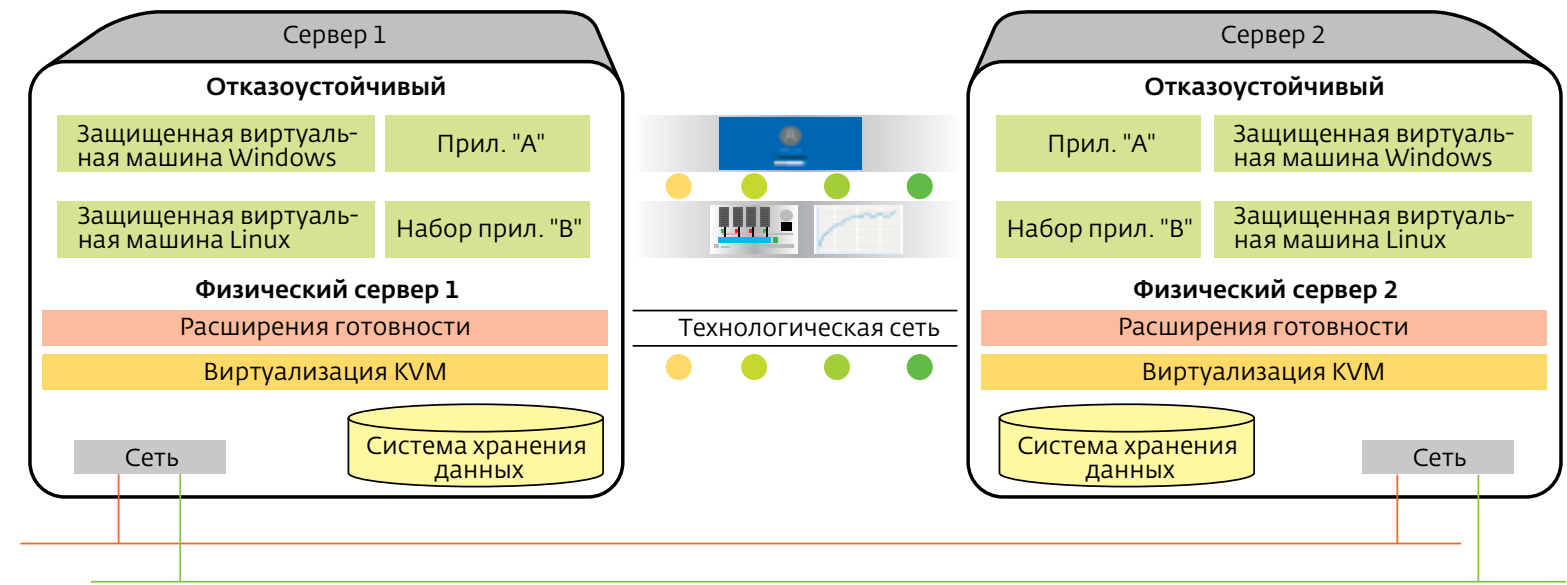

Рис.7. Упрощенная схема вычислительного комплекса повышенной надежности и живучести под управлением ПО Stratus everRun
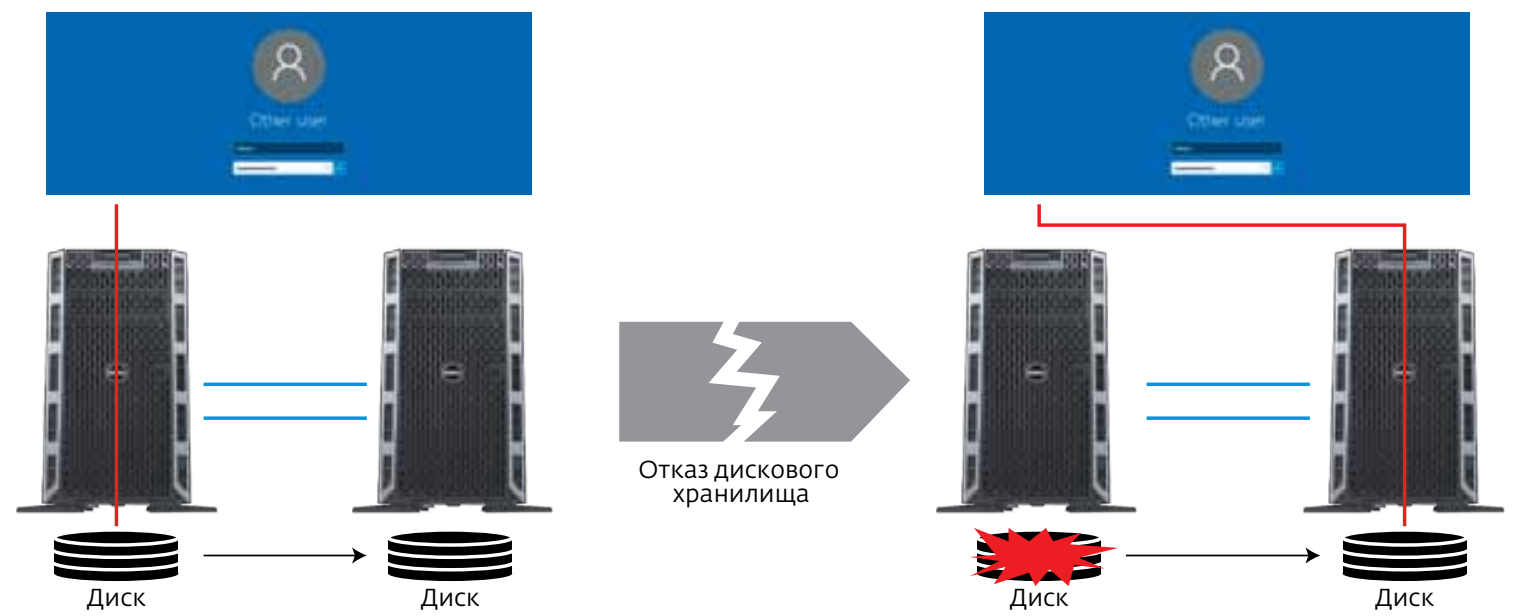

Рис.8. Схема реализации ИС второго уровня отказоустойчивости (при выходе из строя устройства внешней памяти)

и приложения загружаются на другом узле. На втором уровне при выходе из строя компонентов (жесткого диска или сетевой платы), защищаемая ОС использует аналогичный ресурс на втором узле, время простоя при этом равно нулю. Схема реализации ИС второго уровня отказоустойчивости при выходе из строя устройства внешней памяти представлена на рис.8.
При выходе из строя одного из узлов целиком защищаемая ОС загружается на втором узле. Доступность сервера приостанавливается только на время загрузки ОС на этом узле. Схема реализации ИС второго уровня отказоустойчивости при выходе из строя вычислительного узла представлена на рис.9.

На третьем уровне при выходе из строя узла целиком, либо его компонентов, защищаемая 

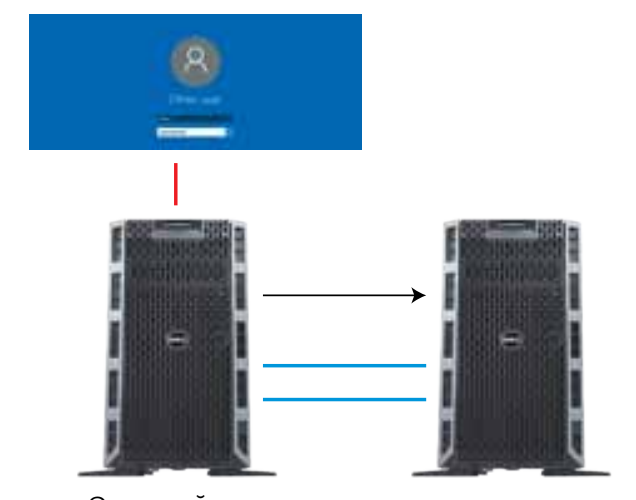

Основной узел функционирует нормально

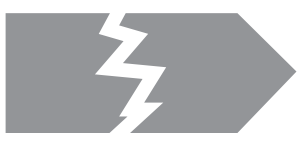

сбой системы

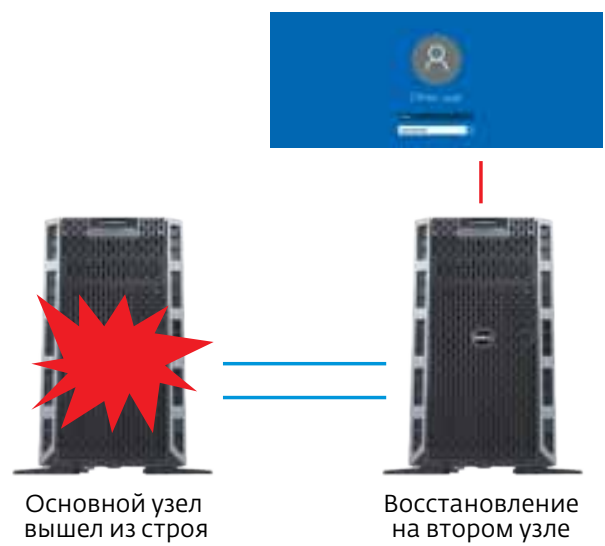

Рис.9. Схема реализации ИС второго уровня отказоустойчивости (при выходе из строя вычислительного узла)

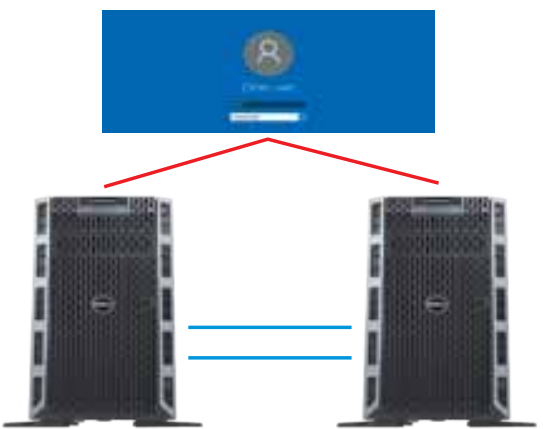

Оба узла

функционируют нормально
Отказ сервера

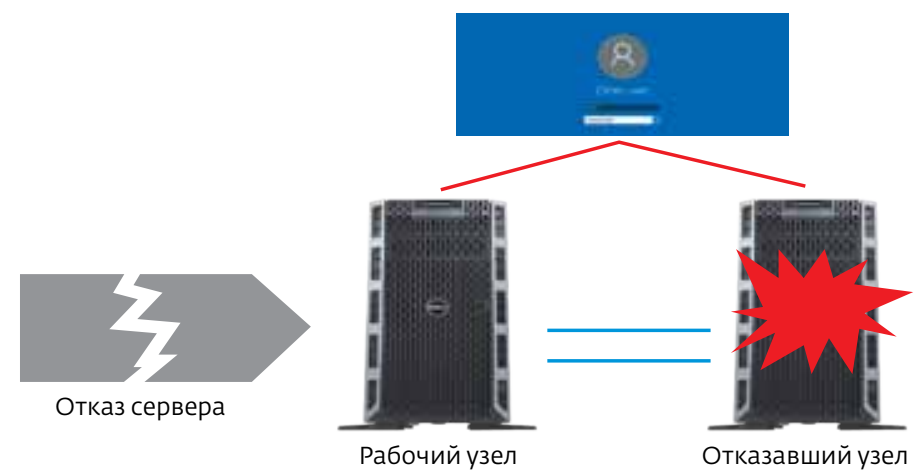

Рис.10. Схема реализации ИС третьего уровня отказоустойчивости (при выходе из строя вычислительного узла)

OC всегда остается доступной для пользователей. Время простоя фактически равно нулю. Схема реализации ИС третьего уровня отказоустойчивости представлена на рис.10. На втором и третьем уровнях допускается отключение одного из вычислительных узлов системы для обслуживания без потери доступности виртуального сервера.

Структурная схема БС повышенной надежности и живучести на оборудовании Viper-SC+ base station (140-5318-502) с подключением к серверу третьего уровня отказоустойчивости и не имеющей единой точки отказа продемонстрирована на рис.11.

Для многих приложений представленная выше интегрированная схема реализации БС и вычислительного комплекса может показаться неоправданно дорогостоящей. Однако, в большинстве случаев затраты на ее внедрение и сопровождение оказываются существенно ниже того ущерба, который может возникнуть в результате сбоя в работе критически важного объекта. 


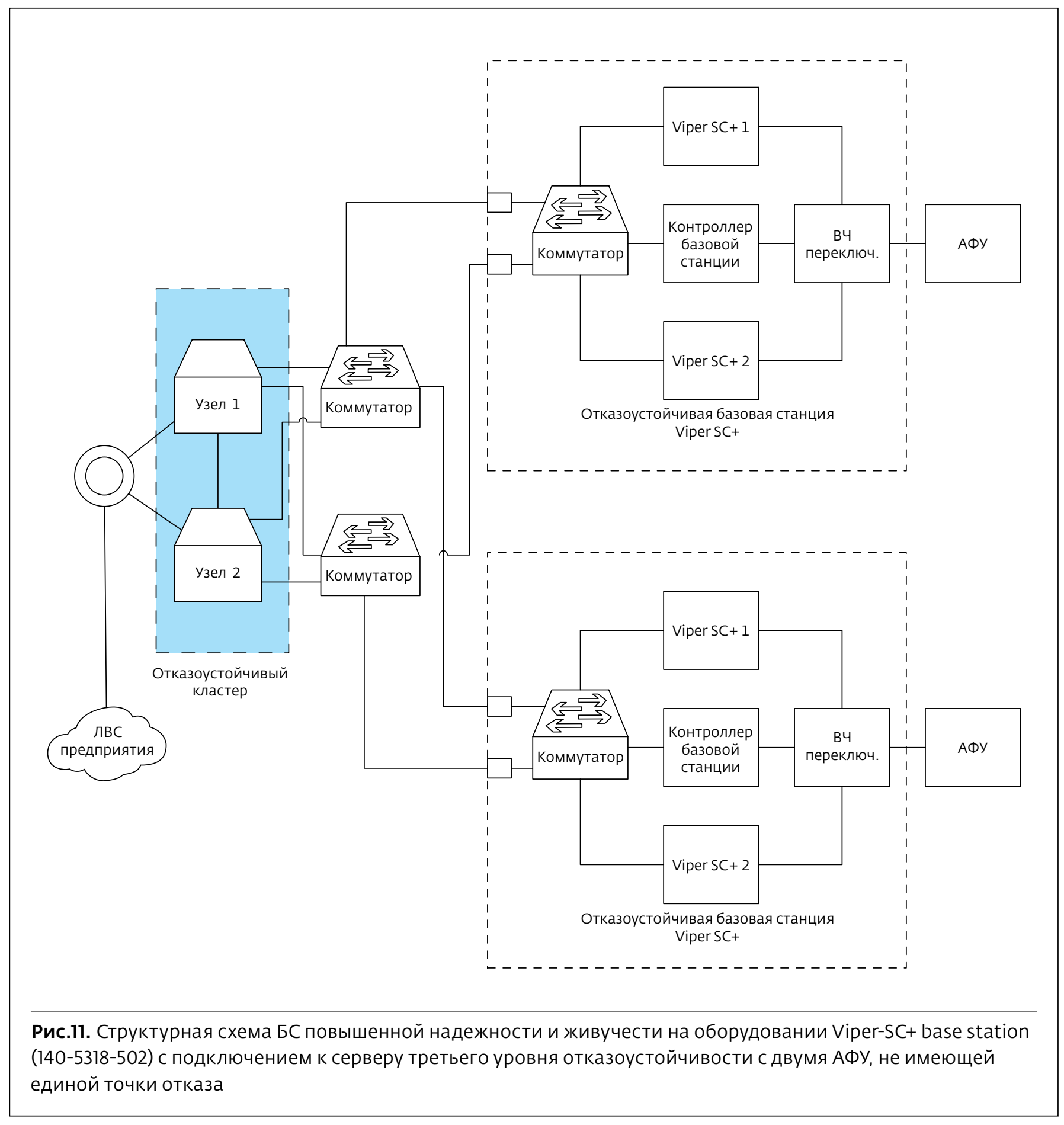

Таким образом, радиотехническая платформа Viper-SC+ в совокупности с программным обеспечением мониторинга технического состояния радиосети и специальным ПО Stratus everRun позволяет строить современные интегрирован ${ }^{-}$ ные, масштабируемые в части надежности и живучести узкополосные технологические радиосети, которые могут эффективно применяться в ответственных системах. Возможности данного решения в части надежности и живучести в полной мере удовлетворяют самым жестким требованиям, предъявляемым действующими руководящими документами, регулирующими эксплуатацию информационного и телекоммуникационного оборудования на критически важных объектах.

\section{ЛИТЕРАТУРА:}

1. Маргарян С. Радиомодемы УКВ-диапазона на интеллектуальном железнодорожном транспорте // ПЕРВАЯ МИЛЯ. 2016. № 4. С. 64-75. 